PROCEEDINGS OF THE

AMERICAN MATHEMATICAL SOCIETY

Volume 134, Number 6, Pages 1701-1705

S 0002-9939(05)08182-7

Article electronically published on December 2, 2005

\title{
ISOMETRIES OF THE DIRICHLET SPACE AMONG THE COMPOSITION OPERATORS
}

\author{
MARÍA J. MARTÍN AND DRAGAN VUKOTIĆ
}

(Communicated by Joseph A. Ball)

\begin{abstract}
We show that every composition operator which is an isometry of the Dirichlet space is induced by a univalent full map of the disk into itself that fixes the origin. This is an analogue of the Hardy space result for inner functions due to Nordgren. The proof relies on the Stone-Weierstrass theorem and the Riesz representation theorem.
\end{abstract}

The Dirichlet space $\mathcal{D}$ of all holomorphic functions with square integrable derivative in the unit disk $\mathbb{D}$ is a distinguished member of a scale of conformally invariant Banach spaces on which the Möbius group acts boundedly; see 1, 2. This scale contains, for example, all analytic Besov spaces $B^{p}, 1 \leq p<\infty$, the Bloch space $\mathcal{B}$, and the little Bloch space $\mathcal{B}_{0}$. Actually, $\mathcal{D}$ is the only Hilbert space among such spaces up to an isomorphism [1. Cima and Wogen [3] characterized the linear isometries of $\mathcal{B}_{0}$ and the isometries of $\mathcal{B}$ that are onto. Hornor and Jamison [7] described all surjective isometries of $B^{p}$ spaces, except when $p=2$ (the Dirichlet space). Similar results are well known for Bergman and Hardy spaces $([5, \S 2.8)$.

Being a Hilbert space, $\mathcal{D}$ has plenty of isometries. They can be obtained, e.g., by permuting elements of an orthonormal basis. Even among the composition operators there are enough isometries of $\mathcal{D}$. In this note we describe all such operators by showing that their symbols must be univalent, fix the origin, and map $\mathbb{D}$ onto a subset of full area measure. This provides an analogue of a known result for the classical Hardy space $H^{2}$. Namely, it was shown by Nordgren ([9, p. 444) that if $\varphi$ is an inner function and $\varphi(0)=0$, then $C_{\varphi}$ is an isometry of $H^{2}$ (see also p. 321 of [4]). The converse is also true (and even a stronger result can be found in [11). Namely, it is not difficult to see that if $C_{\varphi}$ is an isometry, then $\varphi(0)=0$; this can be deduced either from the norm estimate (Corollary 3.7 of [4]), $\left(1-|\varphi(0)|^{2}\right)^{-1 / 2} \leq\left\|C_{\varphi}\right\|=1$, or from the basic property, $C_{\varphi}^{*} C_{\varphi}=I$. The identity function $f(z)=z$ has norm one, hence $\|\varphi\|_{H^{2}}=\left\|C_{\varphi} f\right\|_{H^{2}}=1$. Denoting by $\mathbb{T}$ the unit circle, by $d m=(2 \pi)^{-1} d \theta$ the normalized arc length measure on it, and by $\widetilde{\varphi}$ the radial limit function of $\varphi$ on $\mathbb{T}$, we get $0=\int_{\mathbb{T}}\left(1-|\widetilde{\varphi}|^{2}\right) d m$. Since $|\widetilde{\varphi}| \leq 1$ $m$-a.e., it follows that actually $|\widetilde{\varphi}|=1$ a.e. on $\mathbb{T}$, meaning that $\varphi$ is inner.

Received by the editors December 14, 2004 and, in revised form, January 10, 2005.

2000 Mathematics Subject Classification. Primary 47B33, 31C25.

Both authors were supported by MCyT grant BFM2003-07294-C02-01, Spain.

(C)2005 American Mathematical Society Reverts to public domain 28 years from publication 


\section{BASIC DEFINITIONS AND FACTS}

Recall that a linear isometry of a Banach space is a linear operator $T$ such that $\|T f\|=\|f\|$ for all $f$ in the space. On a Hilbert space this is equivalent to $T^{*} T=I$; if the (Hilbert space) isometry is also onto, it is called a unitary operator and is characterized by the property $T^{*} T=T T^{*}=I$.

The Dirichlet space $\mathcal{D}$ is the set of all analytic functions in the unit disk $\mathbb{D}$ with the finite Dirichlet integral $\int_{\mathbb{D}}\left|f^{\prime}\right|^{2} d A$, where $d A$ denotes the normalized area measure. It is a Hilbert space when equipped with the inner product

$$
\langle f, g\rangle_{\mathcal{D}}=f(0) \overline{g(0)}+\int_{\mathbb{D}} f^{\prime}(z) \overline{g^{\prime}(z)} d A(z)=a_{0} \overline{b_{0}}+\sum_{n=1}^{\infty} n a_{n} \overline{b_{n}},
$$

where $f(z)=\sum_{n=0}^{\infty} a_{n} z^{n}, g(z)=\sum_{n=0}^{\infty} b_{n} z^{n}$. Choosing a suitable analytic branch of the logarithm, one gets the following reproducing kernel:

$$
K_{w}(z)=1+\log \frac{1}{1-\bar{w} z}
$$

meaning that $\left\langle f, K_{w}\right\rangle_{\mathcal{D}}=f(w)$ for all $f$ in $\mathcal{D}$ and all points $w$ in $\mathbb{D}$.

By a holomorphic self-map of the unit disk $\mathbb{D}$ we mean an analytic function $\varphi$ in $\mathbb{D}$ such that $\varphi(\mathbb{D}) \subset \mathbb{D}$. The composition operator with symbol $\varphi$ is defined by $C_{\varphi} f=f \circ \varphi$. By an easy application of the Closed Graph Theorem, its mere action on any classical Banach or Hilbert space of analytic functions in the disk implies its boundedness on that space. Two excellent sources for the theory of such operators are [10] and [4].

While $C_{\varphi}$ is bounded on any Hardy or Bergman space for every possible symbol $\varphi$, this is not automatic on $\mathcal{D}$. A necessary and sufficient condition for the boundedness of $C_{\varphi}$ there can be stated as a Carleson measure condition (as was first shown in Voas' unpublished thesis [12]; see also Theorem 13 of [2]):

$$
\int_{S(\zeta, h)} n_{\varphi} d A \leq C \int_{S(\zeta, h)} d A=K h^{2}
$$

for all $\zeta \in \partial \mathbb{D}$, and all $h \in(0,1)$. Here $S(\zeta, h)=\{z \in \mathbb{D}:|z-\zeta|<h\}$ is a typical Carleson set. Also, as is usual, $n_{\varphi}(w), w \in \mathbb{D}$, denotes the counting function, defined as the cardinality of the set $\{z \in \mathbb{D}: \varphi(z)=w\}$ when the latter is finite and understood as the symbol $\infty$ otherwise, with the usual rules of arithmetic holding in relation to the Lebesgue integral.

The reader should be warned that the Dirichlet space defined in the standard reference 44 (from p. 14 on) and the one considered both here and in the recent relevant paper [6] are equal as sets but are only isomorphic as Hilbert spaces. They are certainly not isometric, and their reproducing kernels are slightly different. This should affect the norm properties of composition operators although usually there do not seem to exist significant differences in the two resulting theories.

Recall that a self-map of $\mathbb{D}$ is said to be a univalent full map if it is one-to-one and $A[\mathbb{D} \backslash \varphi(\mathbb{D})]=0$. An example is a conformal map onto the disk minus finitely or countably many disjoint slits. Full mappings are well-studied objects in the theory of univalent functions, for example. Univalent full maps of the disk induce bounded composition operators on the Dirichlet space. They came up quite naturally in our recent work on norm computations [8], and here they appear again. 


\section{Proof of the main Result}

It is a known fact that, among the composition operators, the only unitary operators are the rotations, both on $H^{2}$ and on $\mathcal{D}$. We refer the reader to Section 3 of [6] for the Dirichlet space defined in the same way as in the present note and to pp. 309-312 of 4 for a more general statement for the alternative definition of $\mathcal{D}$. We include a very short proof of this fact that is independent of our main result.

Proposition. Suppose $C_{\varphi}$ is bounded on $\mathcal{D}$. Then it is a unitary operator if and only if $\varphi(z)=\lambda z$, where $|\lambda|=1$.

Proof. It is well known and simple to check that $C_{\varphi}^{*} K_{w}=K_{\varphi(w)}$ for all $w$ in $\mathbb{D}$. Assuming $C_{\varphi}$ is unitary, we have $C_{\varphi} C_{\varphi}^{*}=I$, hence $C_{\varphi} C_{\varphi}^{*} K_{w}=K_{w}$ so that $K_{\varphi(w)}(\varphi(z))=K_{w}(z)$ for all $z, w$ in $\mathbb{D}$. Since the function $\log 1 /(1-\zeta)$ is univalent in $\mathbb{D}$, it follows from the definition of $K_{w}$ that $\overline{\varphi(w)} \varphi(z)=\bar{w} z$ for all $z, w$ in $\mathbb{D}$. Thus, $\varphi(0)=0$ and $|\varphi(z) / z|=$ const in $\mathbb{D}$. By elementary properties of analytic functions, $\varphi(z)=\lambda z$ for some constant $\lambda$. Going back to an earlier identity, this means that $\bar{w} z=|\lambda|^{2} \bar{w} z$, hence $|\lambda|=1$.

It is easily verified that $C_{\varphi}$ is a surjective isometry for every rotation $\varphi$.

What other symbols can induce an isometry of the Dirichlet space? By a simple change of variable $w=\varphi(z)$, a composition operator $C_{\varphi}$ induced by any univalent full map $\varphi$ of $\mathbb{D}$ such that $\varphi(0)=0$ is an isometry on $\mathcal{D}$ :

$$
\left\|C_{\varphi} f\right\|_{\mathcal{D}}^{2}=|f(\varphi(0))|^{2}+\int_{\mathbb{D}}\left|(f \circ \varphi)^{\prime}\right|^{2} d A=|f(0)|^{2}+\int_{\varphi(\mathbb{D})}\left|f^{\prime}\right|^{2} d A=\|f\|_{\mathcal{D}}^{2} .
$$

It is natural to ask whether there are any other isometries among the composition operators acting on $\mathcal{D}$. The result below asserts that there are none. It can, thus, be interpreted as follows: the univalent full maps of the disk that fix the origin are the Dirichlet space counterparts of the inner functions that fix the origin for the composition operators on $H^{2}$.

Theorem. A composition operator $C_{\varphi}$ that acts on $\mathcal{D}$ is an isometry if and only if $\varphi$ is a univalent full map of $\mathbb{D}$ that fixes the origin.

Proof. Let $C_{\varphi}$ be an isometry of $\mathcal{D}$. Observe first that $\varphi(0)=0$. This follows from

$$
f(0)=\langle f, 1\rangle_{\mathcal{D}}=\left\langle C_{\varphi}^{*} C_{\varphi} f, 1\right\rangle_{\mathcal{D}}=\left\langle C_{\varphi} f, C_{\varphi} 1\right\rangle_{\mathcal{D}}=\left\langle C_{\varphi} f, 1\right\rangle_{\mathcal{D}}=f(\varphi(0))
$$

by choosing $f(z)=z$. Also, since $C_{\varphi}$ is an isometry we have by the Area Formula (change of variable) from p. 36 of [4] that

$$
\int_{\mathbb{D}}\left|f^{\prime}\right|^{2} d A=\int_{\mathbb{D}}\left|f^{\prime} \circ \varphi\right|^{2}\left|\varphi^{\prime}\right|^{2} d A=\int_{\mathbb{D}}\left|f^{\prime}\right|^{2} n_{\varphi} d A, \quad f \in \mathcal{D} .
$$

That is, for every function $g$ in the Bergman space $A^{2}$ of the square integrable analytic functions in $\mathbb{D}$ we have

$$
\int_{\mathbb{D}}|g|^{2} d A=\int_{\mathbb{D}}|g|^{2} n_{\varphi} d A .
$$

This means that the standard Bergman space $A^{2}$ and the weighted Bergman space $A^{2}\left(n_{\varphi} d A\right)$ coincide as sets, and the identity map is an isometry between these two 
inner product spaces. The respective polarization identities yield

$$
\int_{\mathbb{D}} f \bar{g} d A=\int_{\mathbb{D}} f \bar{g} n_{\varphi} d A, \quad f, g \in A^{2}
$$

In particular, choosing $f(z)=z^{m}, m \geq 0$, and $g(z)=z^{n}, n \geq 0$, we get that

$$
\int_{\mathbb{D}} P(z, \bar{z}) d A=\int_{\mathbb{D}} P(z, \bar{z}) n_{\varphi} d A,
$$

for all polynomials $P$ in $z$ and $\bar{z}$. The Stone-Weierstrass theorem now yields

$$
\int_{\mathbb{D}} u d A=\int_{\mathbb{D}} u n_{\varphi} d A
$$

for all functions $u \in C(\overline{\mathbb{D}})$. Finally, the Riesz representation theorem implies that $d A=n_{\varphi} d A$, that is, $n_{\varphi}=1$ almost everywhere on $\mathbb{D}$ with respect to $d A$. Thus, $A[\mathbb{D} \backslash \varphi(\mathbb{D})]=0$.

It is only left to check that if $n_{\varphi}=1$ almost everywhere, then $\varphi$ must be univalent in all of $\mathbb{D}$. This follows by an application of the open mapping theorem for analytic functions. Namely, assuming that $\varphi(a)=\varphi(b)=c$ for two distinct points $a, b \in \mathbb{D}$, we can choose two disjoint disks $D_{a}$ and $D_{b}$ centered at these points, respectively, and observe that their images under $\varphi$ intersect. Hence there is an open disk in $\varphi(\mathbb{D})$ that contains $c$ and which is the image under $\varphi$ of an open subset of $D_{a}$ and of an open subset of $D_{b}$. Note that for every $z$ in this disk, $n_{\varphi}(z)$ is at least 2, contradicting $n_{\varphi}=1$ a.e. on $\mathbb{D}$. This completes the proof.

\section{ACKNOWLEDGMENT}

The authors thank Manuel Contreras for pointing out several misprints in the first draft of the manuscript and the referee for helpful comments and suggestions that improved the exposition.

\section{REFERENCES}

[1] J. Arazy and S.D. Fisher, The uniqueness of the Dirichlet space among Möbius-invariant Hilbert spaces, Illinois J. Math. 29 (1985), no. 3, 449-462. MR0786732 (86j:30072)

[2] J. Arazy, S.D. Fisher, and J. Peetre, Möbius invariant function spaces, J. Reine Angew. Math. 363 (1985), 110-145. MR0814017 (87f:30104)

[3] J.A. Cima and W.R. Wogen, On isometries of the Bloch space, Illinois J. Math. 24 (1980), no. 2, 313-316. MR0575069 (82m:30052)

[4] C. Cowen and B. MacCluer, Composition Operators on Spaces of Analytic Functions, Studies in Advanced Mathematics, CRC Press, Boca Raton, 1995. MR1397026 (97i:47056)

[5] P.L. Duren and A.P. Schuster, Bergman Spaces, Mathematical Surveys and Monographs 100, American Mathematical Society, Providence, RI, 2004. MR2033762 (2005c:30053)

[6] E.A. Gallardo-Gutiérrez and A. Montes-Rodríguez, Adjoints of linear fractional compostion operators on the Dirichlet space, Math. Ann., 327 (2003), no. 1, 117-134. MR2005124 (2004h:47036)

[7] W. Hornor and J.E. Jamison, Isometries of some Banach spaces of analytic functions, Integral Equations Operator Theory 41 (2001), no. 4, 410-425. MR1857800(2002h:46035)

[8] M.J. Martín and D. Vukotić, Norms and spectral radii of composition operators acting on the Dirichlet space, J. Math. Anal. Appl. 304 (2005), 22-32. MR2124646

[9] E.A. Nordgren, Composition operators, Canad. J. Math. 20 (1968), 442-449. MR0223914 $(36: 6961)$

[10] J.H. Shapiro, Composition Operators and Classical Function Theory, Springer-Verlag, New York, 1993. MR1237406 (94k:47049) 
[11] J.H. Shapiro, What do composition operators know about inner functions?, Monatsh. Math. 130 (2000), 57-70. MR.1762064 (2001a:47029)

[12] C. Voas, Toeplitz Operators and Univalent Functions, Ph.D. Thesis, University of Virginia, 1980.

Departamento de Economía, Universidad Carlos iII de Madrid, Calle Madrid 126, 28903 Getafe (Madrid), Spain

E-mail address: mjmartin@eco.uc3m.es

Current address: Departamento de Matemáticas, Universidad Autónoma de Madrid, 28049 Madrid, Spain

E-mail address: mjose.martin@uam.es

Departamento de Matemáticas, Universidad Autónoma de Madrid, 28049 Madrid, SPAIN

E-mail address: dragan.vukotic@uam.es 